\title{
PERFORMANCE OF A HIGH DENSITY PROJECTION CHAMBER
}

\author{
M. BERGGREN *, A. CATTAI **, H.G. FISCHER, P.S. IVERSEN ***, E. LILLETHUN ***, \\ M. PANTER, O. ULLALAND and J.R. URSIN
}

CERN, Geneva, Switzerland

\begin{abstract}
The high density projection chamber (HPC) is a large volume gas sampling calorimeter. The highly granulated module allows a complete three-dimensional reconstruction of the shower and offers good energy resolution. We will present preliminary results from test runs and discuss operation in a magnetic field.
\end{abstract}

\section{Introduction}

The new high energy physics experiments are emphasizing the use of large calorimeter systems. These systems have to cover the full solid angle and enable particle separation within a jet-like event structure. There are several advantages to a projection chamber approach in sampling calorimeters. It gives good energy resolution, together with a complete representation of the energy flow. This gives the calorimeter a fine tracking capability and enhances the possibility to separate electrons and hadrons, and single gammas from $\pi^{0}$ decay. The HPC is shown in fig. 1.

The HPC is a conceptual now approach to sampling calorimeters. It has therefore been necessary to prove the feasibility of this detector with prototype work. The construction method and early tests have already been published [1-3].

The HPC is separated in a converter/drift structure and a single plane active detector. The converter forms a large volume gas sampling structure which is mechanically self-stable. The primary ionization from the shower is then drifted along the sampling slots to a proportional wire chamber. This technique results in an inherent resolution of $\sim 500 \mu \mathrm{m}$ transverse to the shower axis and one sampling slot along the shower.

We will in this paper discuss results for the energy resolution obtained with an electron beam and show the influence of a magnetic field on this resolution. We shall first describe charge drift in the sampling slots and compare it to other geometry.

* University of Stockholm, Stockholm, Sweden.

** INFN Sanita, Rome, Italy.

*** University of Bergen, Bergen, Norway.

\section{Charge transport}

The attenuation length for this detector is given by the ability to drift electrons in confined channels. The drift channels are made by superimposing grid struc-

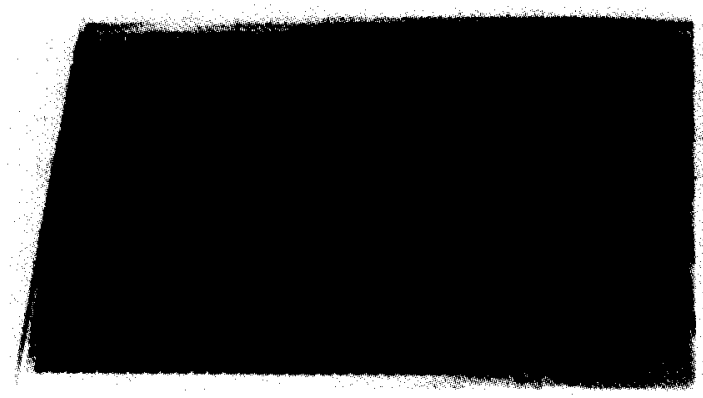

CATHODE

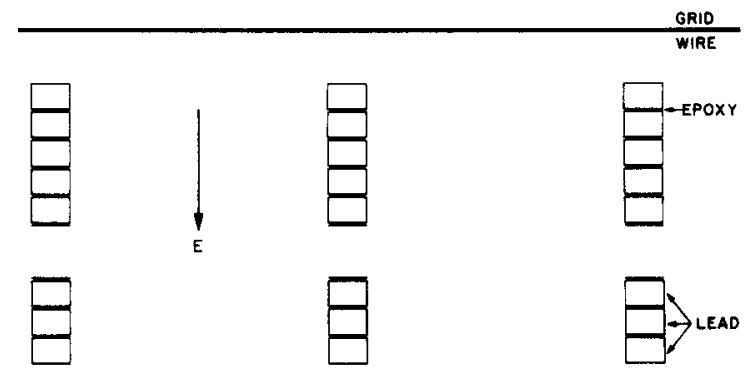

Fig. 1. (a) A close-up of the converter module for the HPC; (b) layout of the drift structure and the pick-up chamber.

II. CALORIMETRY 
tured metal plates. For the test module, we used $1 \mathrm{~mm}$ thick lead plates. The drift slots were $10 \mathrm{~mm}$ wide and the converter bars were $1.4 \mathrm{~mm}$ deep. Each plate has a $50 \mu \mathrm{m}$ epoxy layer on each side, which acts as an electrical insulator and a bonding agent between each plate (fig. 1). In this way, we obtain a mechanically good laminate, which only needs a light support structure to be stable [3]. The drift field is made by connecting each plate to an external voltage divider.

The effective gas layer of the sampling slots will be smaller than the geometrical dimension, due to electric field inhomogeneities next to the walls $[1,3]$. This effect can, however, be neglected when the ratio between the slot width and the plate thickness is larger than 8. In this case, all the charge losses can be attributed to transverse diffusion in the gas.

We have measured the charge attenuation length in a $1 \mathrm{~m}$ long drift module. The electric drift field is produced by a pattern of copper strips, $1 \mathrm{~mm}$ wide, on a printed circuit board. The distance between each strip is $100 \mu \mathrm{m}$ and the drift channel is $7 \mathrm{~mm}$ wide. Perpendicular to the drift axis it is possible to introduce a $\gamma$ or an $\alpha$ particle and thereby measure the transport property of the channel with respect to electric field strength and gas mixture. The results are given in fig. 2 , together with the predicted attenuation length as a function of the transverse diffusion. Also shown are measurements in a lead laminate structure [4]. The measurements are in agreement with previously published data $[1,5,6]$. The effect of the transverse diffusion in the absence of a

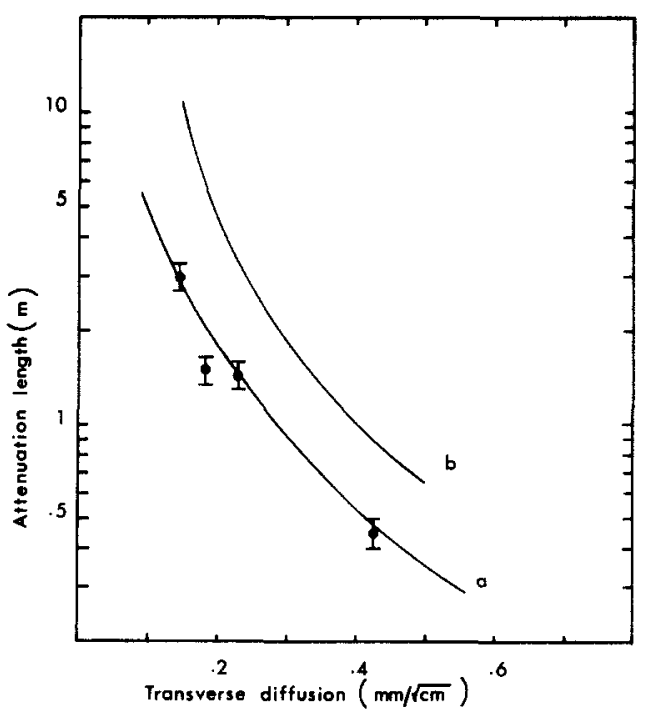

Fig. 2. (a) The charge attenuation length in a $7 \mathrm{~mm}$ wide drift channel as a function of the transverse diffusion in the gas. The line gives the predicted result for this drift structure; (b) predicted attenuation length for the same geometry, but with a $1 \%$ increase of the electric field for each centimetre of drift. magnetic field can be offset by a grading of the electric field [7]. The expected effect of such a field is shown in fig. $2 b$. This shows that the HPC has a good drift structure where the charge losses can be kept small.

Many groups have over the last few years successfully developed sampling calorimeters of the projection chamber type. L.E. Price [7] and C. Gruhn [8] use copper strips on printed circuit boards to set up the drift potential. The interpolation between each voltage step is performed with conductive ink. The resistivity of the ink is $\sim 10^{8}-10^{10} \Omega$ /square. J.E. Cobb [9] uses an extrudable polymer with a few percent of carbon black. This gives an homogeneous drift structure with a bulk resistivity of $-10^{12} \Omega \mathrm{cm}$. All experiments report an attenuation length compatible with prediction. V. Perez-Mendez and collaborators [10], are proposing to use fused lead glass cylinders as the converter structure. Here the resistive layer is produced by heating the $\mathrm{PbO}$ in a hydrogen atmosphere. The resistivity is about $50-200 \mathrm{M} \Omega$ /square.

\section{The test calorimeter}

The HPC test module is shown in fig. 1 . It is 15 radiation lengths deep with 61 gas sampling channels, each $10 \mathrm{~mm}$ wide. The unique feature of the HPC is the three-dimensional readout with only a single active chamber. The chamber has 48 cathode pads. We have developed a complete electronic chain for the test module using a multiplexing integration method [3]. The integration time was $300 \mu \mathrm{s}$, which corresponds to a granularity of $2.6 \mathrm{~mm}$ along drift axis with an electric field of $220 \mathrm{~V} / \mathrm{cm}$ in a gas mixture of $80 \% \mathrm{Ar} / 20 \%$ $\mathrm{CO}_{2}$. The test module is thereby divided into -3000 6 -bit ADC words. The gas amplification was adjusted such that less than $2 \%$ of the non-zero words were in overflow for a $1 \mathrm{GeV}$ shower.

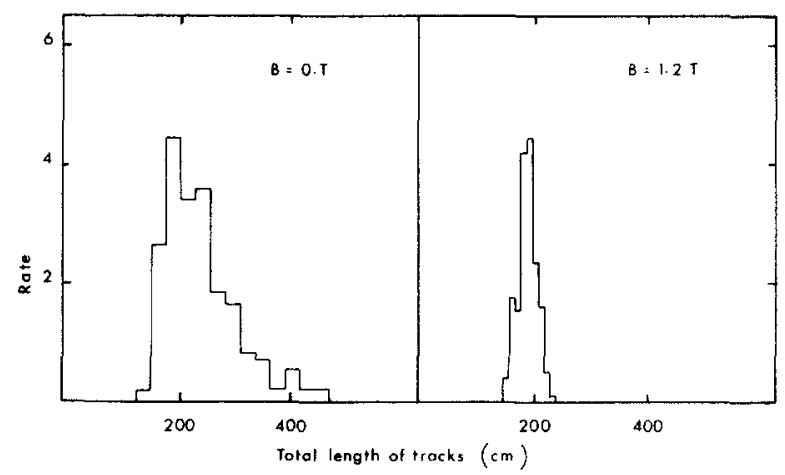

Fig. 3. EGS calculation for the track length/shower with and without a magnetic field. The module used for this calculation had $1.5 \mathrm{~mm}$ of lead between each $7 \mathrm{~mm}$ sampling gap. The shower energy was $1 \mathrm{GeV}$. 


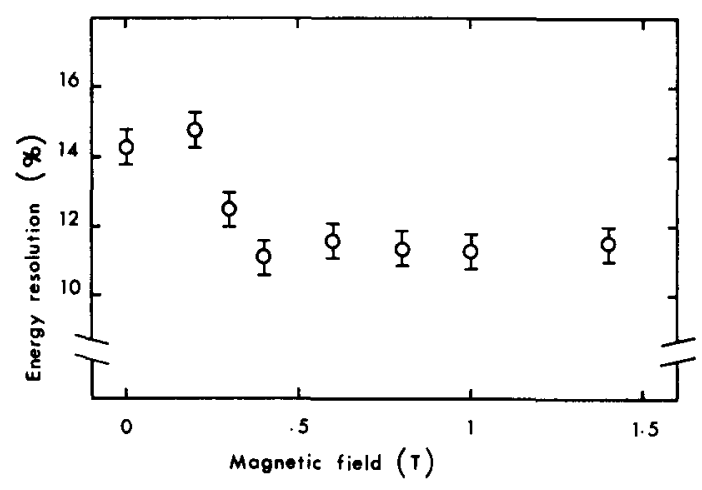

Fig. 4. Measurement of the relative energy resolution as a function of a magnetic field parallel to the drift field.

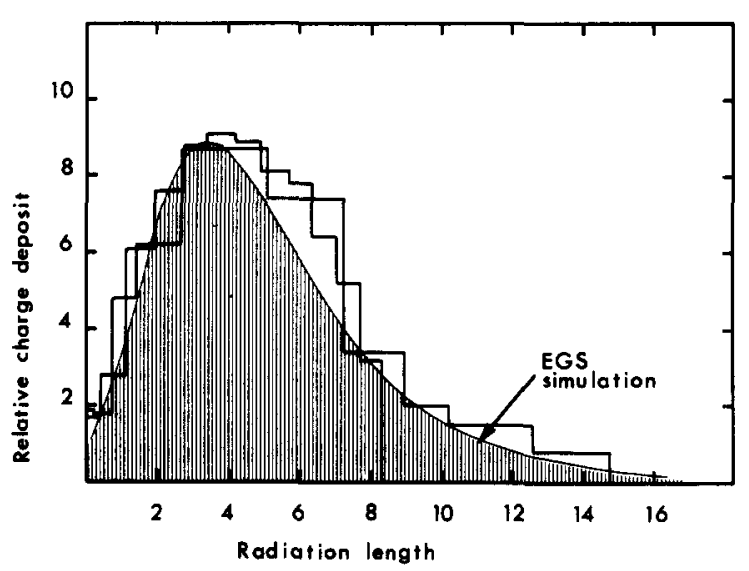

Fig. 5. Longitudinal shower development for a $1 \mathrm{GeV}$ shower. Shown are measurements with an 8 radiation length detector [3] and the results from the present $15 X_{0}$ deep detector together with EGS calculations.
The HPC module was tested at the DESY Electron Synchrotron, with a beam energy from 0.25 to $6 \mathrm{GeV}$. The module was placed inside a magnet which could go up to $1.4 \mathrm{~T}$ with a magnetic field parallel to the electric field.

The energy resolution in a gas sampling calorimeter is a superposition of the sampling fluctuations, the track length fluctuations and the Landau fluctuations. The last two effects contribute with approximately the same strength [11]. In a magnetic field, the low energy shower electrons are swept back into the walls of the converter. Since these electrons cause the track length fluctuations, the energy resolution should improve in a magnetic field (fig. 3).

We have measured the energy resolution as a function of the magnetic field. The preliminary results are given in fig. 4 . We observe a $20 \%$ improvement in the energy resolution. A complete suppression of the track length fluctuations should give $\sim 25 \%$ improvement.

Fig. 5 gives the measured longitudinal shower development together with previous measurements and an EGS calculation [12] for a $1 \mathrm{GeV}$ electron shower. The simulation describes well the build-up of the shower, but due to the energy cut-off of $1 \mathrm{MeV}$, it will underestimate the number of shower particles in the fall-off of the shower.

Fig. 6 shows the energy deposit from a $1 \mathrm{GeV}$ shower. This clearly demonstrates the capability of the HPC to reconstruct in space the energy flow of the shower. The space resolution for the impact point is given in fig. 7.

Fig. 8 gives the preliminary results for the energy resolution of the detector as a function of the electron energy. We have here adjusted for differences in the electronic gain, atmospheric pressure and temperature. An overall normalization with a punch-through $\mu$ signal

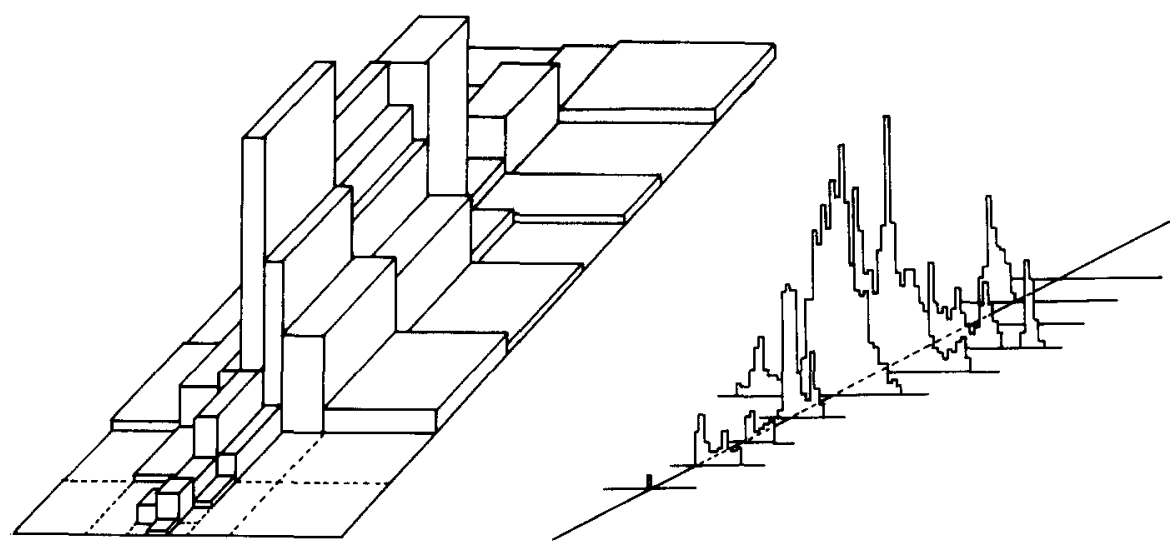

Fig. 6. A single $1 \mathrm{GeV}$ shower in the HPC: (a) total charge deposit on each cathode pad; (b) the shower is projected on the drift time axis for different depths of the detector. 


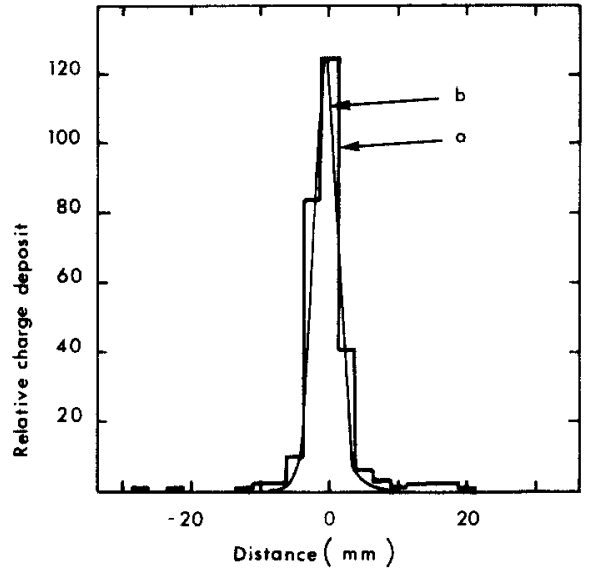

Fig. 7. (a) The charge deposit in the first sampling slot of the HPC for $1 \mathrm{GeV}$ incoming electrons. The beam was $4 \times 4 \mathrm{~mm}^{2}$; (b) the resolution after the beam width has been unfolded.

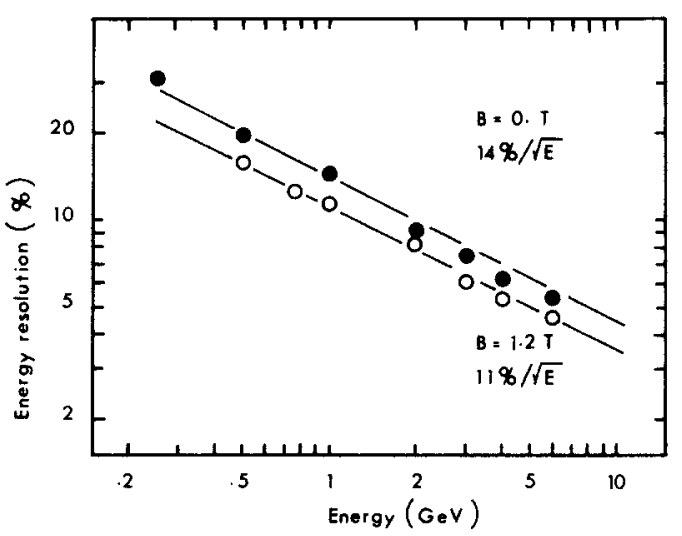

Fig. 8. The relative energy resolution as a function of the electron energy and a magnetic field parallel to the drift field.

will be performed in the near future. The relative energy resolution is compatible with $14 \% / \sqrt{E}$ and decreasing to $11 \% / \sqrt{E}$ in a magnetic field of $1.2 \mathrm{~T}$ parallel to the electric field. We expect the normalization uncertainty to contribute $1-2 \%$ to the energy resolution.

\section{Conclusion}

The HPC is a powerful calorimeter with an excellent tracking capability and an energy resolution for electrons of $10-12 \% / \sqrt{E}$ with a sampling thickness of $0.25 X_{0}$ of lead. It is a simple and stable large volume detector with few active elements. The full power of this detector can, however, only be obtained through a dedicated reconstruction chain.

We wish to thank our technical staff, E. Albrecht, W. Bell, G. Cerutti, M. Flammier and J.C. Legrand for their continuous collaboration and contributions to the realization of the detector. Specially acknowledged is $M$. Gayot of Université Claude Bernard, Lyon for his work on the hybridization of the preamplifier.

We wish to thank P.G. Innocenti and A. Minten for their active interest and support.

We are indebted to our friends in the DELPHI Collaboration for their help in shower calculations and tests at DESY, R. Ely and M. Tyndel, Rutherford Laboratory, A. Pullia and S. Ragazzi, University of Milano, S. Berglund, University of Stockholm and $\mathrm{J}$. Engler, University of Karlsruhe.

\section{References}

[1] H.G. Fischer and O. Ullaland, IEEE Trans. Nucl. Sci. NS-27 (1980) 38.

[2] E. Albrecht et al., Proc. Int. Conf. on Instrumentation for Colliding Beam 1982, SLAC-250 (1982) 212.

[3] E. Albrecht et al., IEEE Trans. Nucl. Sci. NS-30 (1983) 142.

[4] R. Ely and M. Tyndel, private communication (July 1983).

[5] R.W. Warren and J.H. Parker, Phys. Rev. 188 (1962) 2668.

[6] D. Fancher et al., Nucl. Instr. and Meth. 161 (1979) 383.

[7] L.E. Price, SLAC-250 (1982) 206.

[8] C. Gruhn, private communication (June 1983).

[9] J.E. Cobb, Proc. Int. Conf. on Matter non-conservation, Frascati 1983.

[10] V. Perez-Mendez et al., LBL-15727 (1983), submitted to Nucl. Instr. and Meth.

[11] H.G. Fischer, Nucl. Instr. and Meth. 156 (1978) 81.

[12] R. Ford and W.R. Nelson, SLAC 210 (1978). 